\title{
GoAL-BASED REPRESENTATION IN REPETITIVE Bimanual Movements
}

\author{
Rebecca M. C. Spencer ', Richard B. Ivry' , Eliot Hazeltine², and Andras Semjen ${ }^{3}$ \\ 'University of California, Berkeley, USA \\ ${ }^{2}$ Department of Psychology, University of lowa, USA \\ ${ }^{3}$ Centre National de la Recherche Scientifique, Marseille, France
}

\begin{abstract}
Rhythmic bimanual movements have been the dominant task for understanding coordination involving multiple effectors. This paradigm has led to the development of sophisticated, quantitative models, applicable across a range of situations. However, the role of movement goals during the performance of bimanual movements has received relatively little attention. We review studies implicating the importance of movement goals in rhythmic and non-rhythmic bimanual action. Furthermore, we present a simple experiment that demonstrates how task goals can have a powerful influence on how movement patterns are represented. The placement of real or imagined contact points, or events, was manipulated during in-phase and anti-phase repetitive bimanual wrist movements. Simultaneous vocalizations during the task were used to provide a window into the goal structure associated with the various conditions. The presence of the events resulted in vocalization patterns that were not observed when the tasks were performed in the absence of such events, and the goal structure was dictated by the events rather than the movement pattern. We propose that the manner in which the action is represented is an important constraint underlying the relative stability of coordination of different movement patterns.
\end{abstract}

Key words: coordination, constraints, event-timing

Our daily actions require us to move multiple parts of our body to achieve a single goal. In some cases, the two hands serve a similar role and make similar movements. When we lift a tray, the upward movements of the two hands must be tightly coordinated or the tray will not stay level, spilling its contents. In other cases, the two hands play distinct roles; rather than performing the same movement with both limbs in a synchronized fashion, each hand must perform a unique movement and coordination of these movements is necessary to accomplish the desired outcome. For example, when we spread butter on a piece of bread, one hand must hold and adjust the position of the slice of bread so that the movements of the knife, controlled by the other hand, produce a smooth, even smear.

Correspondence concerning this article should be addressed to Rebecca M. C. Spencer, Department of Psychology, University of California, Berkeley, 3210 Tolman Hall \#1650,Berkeley, CA 94720-1650;tel: 510.642.9226,fax: 510.642.5293,rspencer@socrates.berkeley.edu 
R. M. C. Spencer, R. B. Ivry, E. Hazeltine, \& A. Semjen

The production of rhythmic bimanual movements has been the dominant paradigm for investigating how multiple effectors are coordinated. Participants performing such tasks are instructed to move both hands in a cyclical fashion and the phase or frequency relationship between the two hands is manipulated (. This type of task provides many advantages: It is easy for participants to perform and it offers a variety of task variables that can be manipulated, such as movement amplitude, frequency, and the phase relationship between the two hands. Powerful analytic methods, especially those based on general principles of non-linear dynamics, have been derived from the rich data sets obtained in such studies. Moreover, given that locomotion involves the rhythmic oscillation of effectors, such tasks may tap neural mechanisms that play a fundamental role in motor control.

Studies of repetitive movements have produced detailed, mathematically rigorous models that describe the forms of coordination of the two hands and the stability of such patterns. The models account for the two stable modes of coordination of repetitive bimanual movements, in-phase movements in which the phase difference between the two hands is near $0^{\circ}$, and anti-phase movements in which the phase difference is near $180^{\circ}$. A cardinal finding is that in-phase movements are more stable than anti-phase movements, a result that is consistent with a model based on coupled oscillators.

However, repetitive bimanual tasks differ from many "real world" bimanual actions in that the task is typically not structured in terms of discrete subgoals. Rather, the participant is usually instructed to maintain a particular phase relationship during the repetitive movement over a range of frequencies. In these cases, the action can proceed for several seconds without the progression of goal states. The participant may chose to treat a particular movement state as a goal, but the task constraints do not define discrete events. In contrast, many common bimanual tasks, such as tying shoes, require that the two hands produce distinct trajectories to accomplish a shared goal. In such a complex series of movements, the action is punctuated by the accomplishment of discrete subgoals. These subgoals provide structure to the behavior and likely constrain the manner in which the actions of the two hands are coordinated.

Evidence for the role of goals or external events mediating bimanual coordination has emerged from a number of recent studies. Wiesendanger and colleagues pioneered a drawer-opening task that is demarcated by subgoals. One hand must open the drawer, as the other hand, in parallel, prepares to grasp and lift a peg. The goal structure of the task drives the temporal coordination of the two hands. While the movements of each hand vary considerably from trial to trial, the two hands are closely synchronized as the peg is lifted. This coupling is less apparent during other parts of the movements. For example, the interval between the movement onset of the grasping hand and the onset of the drawer opening is more variable. The temporal goal invariance was preserved when vision was excluded and when somatosensory cues were eliminated with a conduction nerve block of the index finger and thumb of the hand used to open the drawer. Thus, it appears that the hand trajectories are planned with respect to the accomplishment of task-relevant end-states rather than kinematic properties of the separate hands.

Studies of repetitive bimanual movement tasks that include movement subgoals further implicate the importance of the representation of the task goal for understanding interlimb 
coordination. For instance, Byblow and colleagues (Byblow, Carson, \& Goodman, 1994) report that the coordination of bimanual movements is stabilized when the movements are paced by a metronome. Flexion becomes synchronized with the metronome beat and phase variability is minimized at this point in the cycle, an effect referred to as "anchoring." Furthermore, Fink, Foo, Jirsa, and Kelso illustrated that movements can be stabilized if the metronome is presented twice per cycle and participants are instructed to flex and extend concurrent with the tones. Under this double-metronome structure, the anti-phase movements remained stable at high frequencies and transitions to the in-phase pattern were sometimes eliminated.

Neuropsychological studies provide converging evidence of the role of goals in the representation of bimanual movements. Callosotomy patients, individuals whose cerebral hemispheres have been surgically disconnected, show strong temporal coupling on a repetitive bimanual table-tapping task (Eliassen, Baynes, \& Gazzaniga, 2000; Ivry \& Hazeltine, 1999; Tuller \& Kelso, 1989). Furthermore, like neurologically intact controls, temporal variability is reduced in these patients when tapping bimanually compared to when tapping with either hand alone (Ivry \& Hazeltine). This result suggests that the locus of discrete coupling is subcortical; a cortical locus would predict preserved coupling but fails to account for the improvement in consistency during bimanual tapping.

Kennerley, Diedrichsen, Hazeltine, Semien, and Ivry (2002) tested callosotomy patients on a tapping task in which the instructions emphasized that the movements should be either continuous with smooth transitions or discrete with a pause between each cycle. As in previous tasks with event goals, the discrete taps were tightly coupled for both callosotomy patients and control participants. In contrast, the movements of the callosotomy patients became uncoupled in the continuous movement condition. One interpretation of this dissociation is that, despite their superficial similarity, the representation of the task goals is qualitatively different for the discrete and continuous tapping tasks. In the former, salient features such as abrupt reversals, external contacts, or in this case pauses, define events that impose a temporal structure such that each cycle is represented as a unique goal to reproduce the target interval. Given that the callosotomy patients remain coupled in this condition, it is hypothesized that the temporal coordination of the goals is mediated by subcortical neural mechanisms. In contrast, the lack of coupling in the continuous condition suggests that the movement goal is not defined in this manner. The goal of maintaining movement smoothness in this condition may involve a representation in which the spatial trajectory for each hand is continuously modulated. Whereas the interaction between such spatial codes is frequently observed during bimanual movements in normal participants (and thus may account for the preserved coupling for these individuals), the two hands show much greater independence in callosotomy patients (Franz, Eliassen, Ivry, \& Gazzaniga, 1996).

Converging evidence for this hypothesized distinction between the discrete and continuous tapping conditions is provided by a recent study involving unimanual movements. Individuals with cerebellar damage exhibited increased timing variability on a discrete tapping task but not when the instructions emphasized that the movements should be made in a continuous manner. Moreover, these patients are impaired in achieving the appropriate timing of the subgoals in the drawer-opening task (Serrien \& Wiesendanger, 2000). Thus, the cerebellum may provide the signals that demarcate the timing of salient events when task goals require this form of representation. 
R. M. C. Spencer, R. B. Ivry, E. Hazeltine, \& A. Semjen

These examples suggest that constraints on bimanual coordination can be influenced by the structure of the events associated with the task goals. Here we present an experiment designed to investigate whether the representation of task goals during repetitive bimanual movements differs for in-phase and anti-phase conditions. To accomplish this, participants produced inor anti-phase movements by abduction and adduction of the wrist. We manipulated the occurrence of salient events during the movement cycle by varying the location of a contact surface, under the hypothesis that contact of the board by a hand or hands would define temporal events. To capture the manner in which the task goals were represented, participants were instructed to vocalize repeatedly (by saying the word "ba") as they moved their wrists. Importantly, no instructions or demonstrations were given as to the frequency of vocalizations. We assumed that the vocalizations provide insight into the goal representation of the bimanual movements.

By varying the location of the external contacts, we sought to manipulate the representation of the event structure underlying performance. For one of the event conditions, the boards were located between the hands; in the other, the boards were to the right of both hands. When positioned in the center the events, haptic contact with the boards, occurred once per cycle while moving in-phase and contacted twice per cycle when moving anti-phase. When the boards are moved so that both were on the right side of the hands' excursions, the number of external events occurring within a cycle reverses for in- and anti-phase movements; the events occur twice per cycle when moving in-phase and once per cycle when moving anti-phase.

We hypothesize that the manner in which the movements are represented is an important constraint on the stability of bimanual coordination. Contact with the boards is likely to define salient events that will constrain how the task goal is represented. Specifically, the representation of these events would be less complex for in-phase movements when the boards are centered between the hands relative to when the boards are placed to the right of both hands. Conversely, the representation of anti-phase movements would be less complex when the boards are placed to the right of both hands relative to when the boards are placed in the center position.

\section{METHODS}

\section{PARTICIPANTS}

Forty-eight (sixteen male, thirty-two female) right-handed university students aged 18-26 years volunteered for the experiment in exchange for class credit. This research was approved by the Institutional Review Boards of the University of California, Berkeley. Informed consent was obtained from all participants prior to testing.

\section{TASK AND DESIGN}

Participants were seated comfortably at a table. The wrists were oriented with palms down and all movements involved abduction and adduction of the wrists (see Figure 1). To restrict motion to the wrist joint, the participants' forearms were braced to a platform elevated $20 \mathrm{~cm}$ above the table surface.

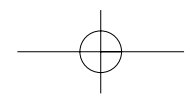




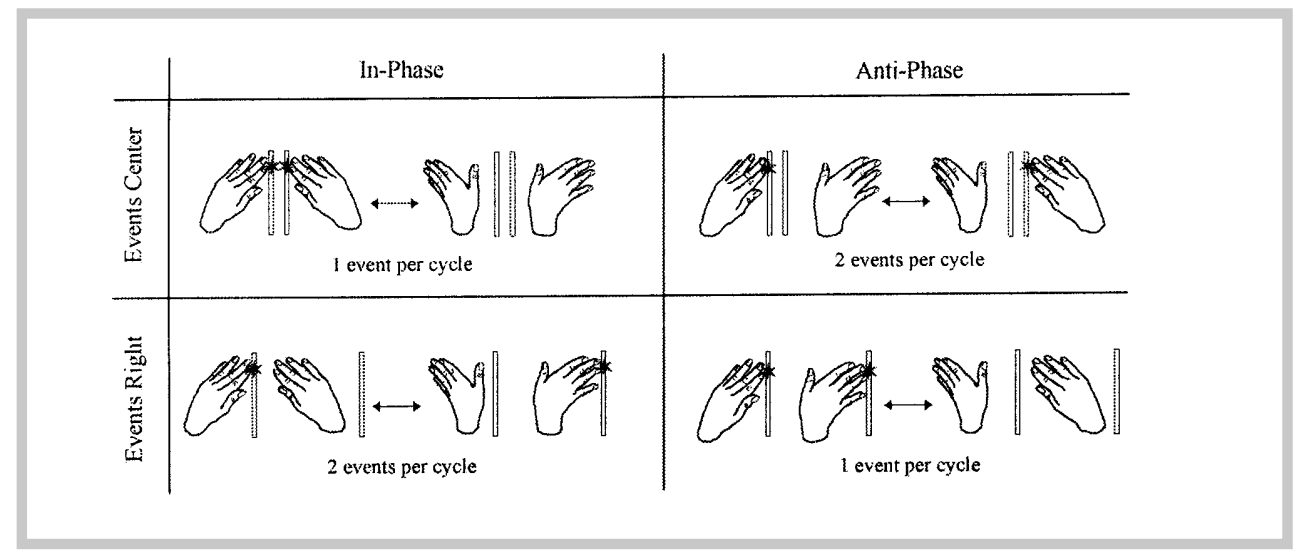

Figure 1. Groups 1 and 2 moved hands in the in-phase coordination mode. Groups 3 and 4 moved in the anti-phase coordination mode. Groups 1 and 3 performed one session with the events in the center and one session with the events to the right of each hand. The * symbol marks an external contact with the wood board.

Participants were randomly assigned to one of four groups. Groups 1 and 2 moved the hands in-phase for all conditions. These participants were instructed to move the hands "in together and out together". Groups 3 and 4 moved in the anti-phase coordination mode. These participants were instructed to move the hands "to the left together and to the right together". Participants were randomly assigned to one of four groups (see Table 1).

Table 1

Summary of conditions

\begin{tabular}{lllll} 
& Group 1 & Group 2 & Group 3 & Group 4 \\
\hline $\begin{array}{l}\text { Coordination } \\
\text { mode }\end{array}$ & in-phase & in-phase & anti-phase & anti-phase \\
\hline Part A* & Events-center & No-events & Events-center & No-events \\
\hline Part B* & Events-right & Imagine-events & Events-right & Imagine-events
\end{tabular}

*Half of the participants performed Part B prior to Part A.

The experiment was divided into two halves. For Groups 1 and 3, wooden boards $\left(8^{\prime \prime} \times 8^{\prime \prime}\right)$ were positioned to provide a contact surface (Figure 1). In one half of the experiment, the two boards were placed between the two hands („Events-center") such that each hand would contact a board when adducted. In the other half of the experiment, the right-hand board was positioned laterally such that contact of the right hand would occur during abduction („Eventsright"). 
R. M. C. Spencer, R. B. Ivry, E. Hazeltine, \& A. Semjen

Groups 2 and 4 performed the tasks without any contact surfaces. In one half of the experiment, the movements were performed without explicit instructions regarding salient events or goals ("No-event"). In the other half, the participants were instructed to "imagine that a wood board was centered between your hands" ("Imagine-events-center")'. Within each group the order of the two halves was balanced.

Participants completed four blocks of each task, with movement speed varied between blocks. For three of these, participants were trained to move such that a hand cycle was completed within $300 \mathrm{~ms}, 500 \mathrm{~ms}$, or $700 \mathrm{~ms}$. For the fourth rate, participants were instructed to adopt a "comfortable" rate. Half of the participants began with the spontaneous rate block, followed by the three specified rates in ascending order. The remaining participants performed the specified-rate blocks in descending order followed by the spontaneous-rate block.

A block consisted of 10 trials; the first five trials contained 10 cycles, the remaining trials contained 30 cycles. After each of the first five trials of specified-rate blocks, verbal feedback was provided indicating the mean cycle duration for that trial. This information was provided to help the participant match the target rate. For the spontaneous-rate blocks, no feedback regarding cycle duration was provided. Following the rate-training trials, participants performed five additional trials in which they were instructed to "Say ' $\mathrm{ba}^{\prime}$ ' repeatedly as you move." Note that we did not use a metronome to specify the hand cycle target rate as the tones could provide an important cue regarding event timing. Furthermore, no demonstration of the movements or vocalizations was provided.

To reduce the likelihood that participants would anticipate our interest in how vocalization rate is influenced by the bimanual movements, between blocks participants were presented with tongue twisters that they were required to repeatedly say for $30 \mathrm{~s}$ ("Bob's big black bath brush broke," "Bobby bopped Betty Boop," or "Betty butters bitter bread").

\section{Data AcQuisition AND ANalysis}

Kinematic data were collected with an Ascension Technology miniBIRD tracking system (138 $\mathrm{Hz}$ sampling rate). One $8 \times 8 \times 12$-mm marker was attached to the index finger of each hand. Cycle durations were computed as the time between local maxima in the $x$-dimension for trajectories produced by the right hand, and the time between local minima in the $x$-dimension for trajectories produced by the left hand.

Vocalizations were recorded with a lapel-microphone and sampled with a digital sound recorder with a sampling rate of $22.05 \mathrm{kHz}$. Voice cycle durations were measured as the time of voice onset to the time of the following voice onset.

\footnotetext{
"We did not include an "imagine events right" condition nor an "imagine no events" condition in this initial study. While
} they would provide a balanced design, we did not feel they provided a critical test of our main predictions.

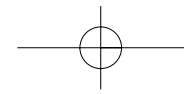




\section{Results}

\section{Hand Gycle Duration}

Mean durations for hand cycle time produced across blocks during trials with simultaneous vocalizations are presented in Figure 2 (cycle duration averaged across hands). Although the participants moved slower than the target duration when the target was $300 \mathrm{~ms}$, the mean cycle durations did not differ significantly between groups.

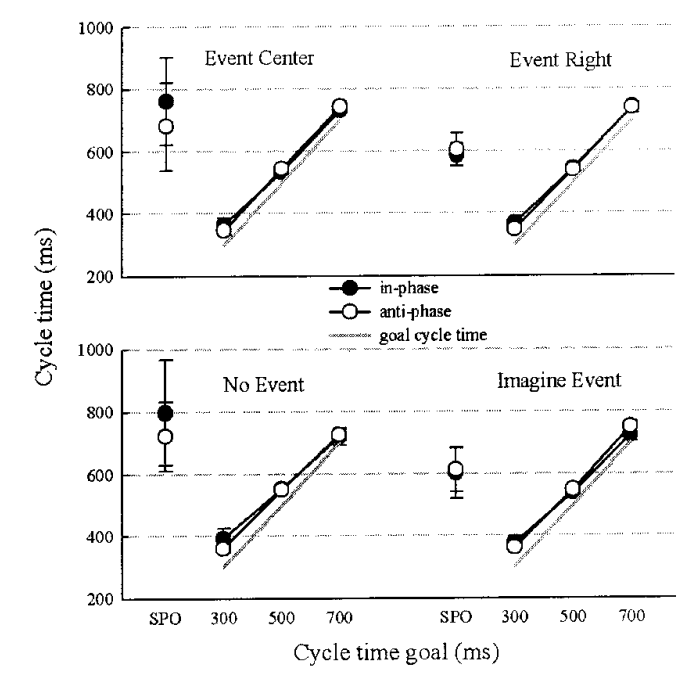

Figure 2. Cycle durations performed across blocks approximated the goal cycle durations.

\section{VOCAL-MANUAL RATIO}

The primary analysis centered on the frequency of "BA" vocalizations, and how this varied as a function of the event timing. In general, there was minimal drift in vocalization rate and movement rate within a trial. Thus, we calculated the ratio of voice cycle time to hand cycle time to reflect the degree of coupling between the vocalizations and movements. Hand and voice cycle durations were computed on a trial by trial basis. Trial averages were then averaged across blocks. It is important to note that all subjects maintained the same general vocal-manual ratio within a block.

To determine the distribution of the ratios, ratios between $.9: 1$ to $1: 1$ were defined as $1: 1$ and ratios between 1.9:1 and 2.1:1 were defined as $2: 1$. The distribution of vocal manual-ratios is presented in Tables 2 and 3. The "Other" category includes performances in which the mean ratio was a simple ratio other than $1: 1$ or $2: 1$ or in which the frequencies of the two actions did not form a simple ratio (i.e., the movements were temporally uncoupled). In fact, the majority of the "Other" category was of the latter type. It is important to emphasize that the relationship between the vocalizations and hand movements remained stable for a given individual within a block. Thus, the high percentage of "Other" is not due to averaging ratios across trials. 
R. M. C. Spencer, R. B. Ivry, E. Hazeltine, \& A. Semjen

The first question centers on whether participants' vocalizations were coupled to their rhythmic hand movements when the instructions do not dictate this constraint and, assuming such coupling occurs, does it differ for in-phase and anti-phase movements. Given the ubiquity of temporal coupling we expected that coupling would be observed in all conditions, reflected by simple ratios.

Table 2

Distribution of vocal-manual ratios for the no-event and imagine-event conditions. Values in parentheses are for participants who performed the task in the first half of the experiment. Values in bold indicate the most frequently chosen ratio category for that task

\begin{tabular}{|c|c|c|c|}
\hline \multirow[b]{2}{*}{$300-\mathrm{ms}$} & \multicolumn{3}{|c|}{ Vocal-manual ratio } \\
\hline & $2: 1$ & $1: 1$ & Other \\
\hline No-event, in-phase & $1(1)$ & $3(2)$ & $8(3)$ \\
\hline No-event, anti-phase & $1(0)$ & $3(1)$ & $8(5)$ \\
\hline Imagine-event, in-phase & $1(0)$ & $3(1)$ & $8(5)$ \\
\hline Imagine-event, anti-phase & $2(0)$ & $3(2)$ & $7(4)$ \\
\hline \multicolumn{4}{|l|}{$500-\mathrm{ms}$} \\
\hline No-event, in-phase & $3(2)$ & $2(1)$ & $7(3)$ \\
\hline No-event, anti-phase & $1(0)$ & $3(0)$ & $8(6)$ \\
\hline Imagine-event, in-phase & $2(1)$ & $3(1)$ & $7(4)$ \\
\hline Imagine-event, anti-phase & $1(1)$ & $2(2)$ & 9 (3) \\
\hline \multicolumn{4}{|l|}{$700-\mathrm{ms}$} \\
\hline No-event, in-phase & $2(1)$ & $3(1)$ & $7(4)$ \\
\hline No-event, anti-phase & $2(1)$ & $3(0)$ & $7(5)$ \\
\hline Imagine-event, in-phase & $2(1)$ & $4(2)$ & $6(3)$ \\
\hline Imagine-event, anti-phase & $2(2)$ & $2(2)$ & $8(2)$ \\
\hline \multicolumn{4}{|l|}{ Spontaneous rate } \\
\hline No-event, in-phase & $2(1)$ & $5(3)$ & $5(2)$ \\
\hline No-event, anti-phase & $2(1)$ & $3(1)$ & $7(4)$ \\
\hline Imagine-event, in-phase & $2(1)$ & $5(3)$ & $5(2)$ \\
\hline Imagine-event, anti-phase & $2(1)$ & $3(3)$ & $7(2)$ \\
\hline
\end{tabular}

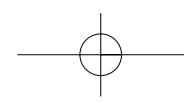


However, in the no-event condition, participants rarely produced simple ratios; the vocalizations and hand movements were temporally uncoupled on most trials (Table 2). In fact, in this condition, most of the participants adopted a single vocalization rate across all hand movement. As illustrated in Figure 3, this resulted in non-simple ratios across all blocks. The results were not influenced by the order in which the tasks were performed. Half of the participants performed the no-event condition after the imagine-event condition. Order did not affect performance, as the distribution of ratios based on all participants was similar to the distribution observed for participants who performed the no-event condition first (Table 2, values in parentheses).

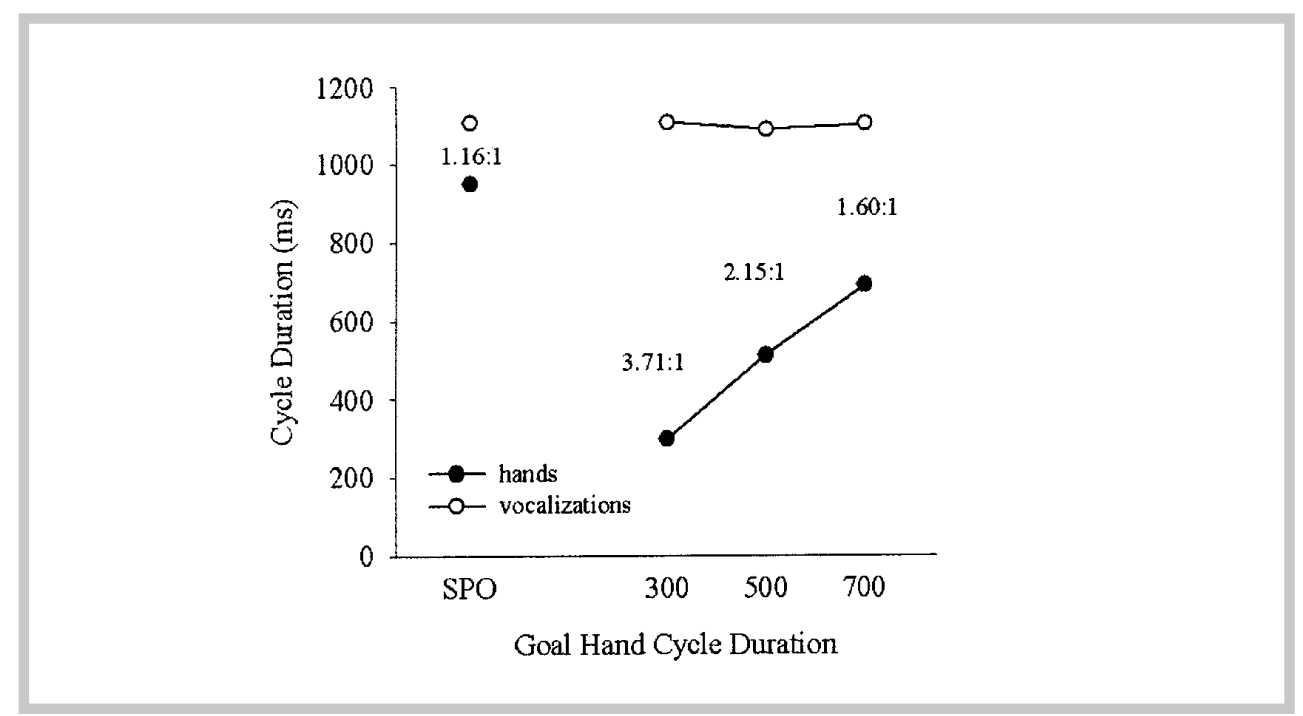

Figure 3. Hand and voice cycle durations produced by one subject in the no-event group. The cycle duration for the vocalizations is relatively constant across the different cycle durations for the hand movements. This invariance produces non-integer ratios of the two actions.

A similar lack of coupling was also evident in the imagine-events-center condition. Again, on most trials, the vocal-manual ratio was not $1: 1$ or $2: 1$. In fact, the slight tendency for participants to adopt a 1:1 ratio in the in-phase condition was reduced when the analysis was restricted to those individuals who performed this condition first. Thus, when participants produced rhythmic abduction/adduction wrist movements without a contact surface, the rate of concurrent vocalizations was relatively independent of the hand movements.

A comparison of the distributions for the conditions without boards, the no-event and imagineevent conditions, emphasized that the participants failed to adopt a consistent frequency relationship between the vocalizations and hand movements. Whether all participants were included or only participants who performed the task first, the percentage of 1:1 and 2:1 ratios was not different between conditions (for all rates $\chi^{2}(6, N=48)<2.32, p>.2$ ), suggesting that imagining the board did not affect the event structure as assayed by the vocalization frequencies. 
R. M. C. Spencer, R. B. Ivry, E. Hazeltine, \& A. Semjen

We next turn to the conditions in which the hands contacted a board on each cycle. We expected the boards would define salient events and thus impose a strong constraint on how the participants represent the temporal structure of the rhythmic hand movements. As seen in Table 3, participants generally adopted simple ratios of $1: 1$ or $2: 1$. Thus, with the contact of the boards, participants were much more likely to synchronize hand and voice cycles than in the conditions in which the board was not present.

\section{Table 3}

Distribution of vocal-manual ratios for the event conditions. Values in parentheses are for participants who performed the task in the first half of the experiment. Values in bold indicate the most frequently chosen ratio category for that task

\begin{tabular}{|c|c|c|c|}
\hline \multirow[b]{2}{*}{ 300-ms } & \multicolumn{3}{|c|}{ Vocal-manual ratio } \\
\hline & $2: 1$ & $1: 1$ & Other \\
\hline Event-right, in-phase & $6(3)$ & $3(2)$ & $3(1)$ \\
\hline Event-right, anti-phase & $2(0)$ & $7(4)$ & $3(2)$ \\
\hline Event-center, in-phase & $3(1)$ & $8(5)$ & $1(0)$ \\
\hline Event-center, anti-phase & $5(5)$ & $3(1)$ & $4(0)$ \\
\hline \multicolumn{4}{|l|}{$500-\mathrm{ms}$} \\
\hline Event-right, in-phase & $6(4)$ & $5(1)$ & $1(1)$ \\
\hline Event-right, anti-phase & $2(1)$ & $7(5)$ & $3(0)$ \\
\hline Event-center, in-phase & $4(1)$ & $7(5)$ & $1(0)$ \\
\hline Event-center, anti-phase & $5(4)$ & $3(1)$ & $4(1)$ \\
\hline \multicolumn{4}{|l|}{ 700-ms } \\
\hline Event-right, in-phase & $8(6)$ & $3(0)$ & $1(0)$ \\
\hline Event-right, anti-phase & $2(2)$ & $8(3)$ & $2(1)$ \\
\hline Event-center, in-phase & $2(1)$ & $8(5)$ & $2(0)$ \\
\hline Event-center, anti-phase & $8(4)$ & $2(1)$ & $2(1)$ \\
\hline \multicolumn{4}{|l|}{ Spontaneous rate } \\
\hline Event-right, in-phase & $6(4)$ & $5(0)$ & $1(2)$ \\
\hline Event-right, anti-phase & $3(0)$ & $6(5)$ & $3(1)$ \\
\hline Event-center, in-phase & $6(1)$ & $6(5)$ & $0(0)$ \\
\hline Event-center, anti-phase & $5(4)$ & $3(1)$ & $4(1)$ \\
\hline
\end{tabular}

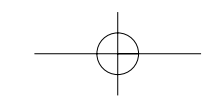


Moreover, if we assume that the number of contacts per cycle defines the event timing of the task, varying the placement of the boards allows us to contrast constraints based on these events with those based on the phase relationship between the two hands. For example, in the boards-right conditions, the two boards are contacted at approximately the same time in the anti-phase condition, defining, we assume, a single event per cycle. In contrast, the boards are contacted in an alternating fashion in the in-phase condition, defining what was assume are two events per cycle. Would the pattern of vocalizations be dictated by the phase requirements or event timing?

The results clearly indicate that the location of the events influenced the distribution of vocalmanual ratios. When data from all participants is considered, the difference between distributions for the events-right and events-center conditions did not reach significance in the $300-\mathrm{ms}\left(\chi^{2}(6, \mathrm{~N}=48)=8.18, \mathrm{p}>1\right), 500-\mathrm{ms}\left(\chi^{2}(6, \mathrm{~N}=48)=7.06, p>1\right)$ and spontaneous-rate $\left(\chi^{2}(6, N=48)=7.40, p>1\right)$ blocks. However the distributions differed significantly in the 700-ms block $\left(\chi^{2}(6, N=48)=13.49, p>.05\right)$. Furthermore, when order effects are eliminated by examining only performances from the first half of the experiment, differences in the distributions are significant for the 300-ms $\left(\chi^{2}(6, N=24)=13.56, p<.05\right)$, $700-\mathrm{ms}\left(\chi^{2}(6, N=24)=13.09, p<.05\right)$, and the spontaneous rate $\left(\chi^{2}(6, N=24)=15.21\right.$, $p<.025)$ blocks. The difference in the distributions of the 500 -ms rate block approached significance $\left(\chi^{2}(6, N=24)=10.93, p<.10\right)$. When the boards were placed in the center, participants exhibited a strong tendency to produce two vocalizations per cycle when moving in the anti-phase coordination mode and one vocalization per cycle during in-phase movements. This pattern was reversed when the right-hand board was shifted lateral to the right hand providing a contact point during abduction. Here in-phase movements were associated with two vocalizations per cycle and anti-phase movements with one vocalization per cycle.

\section{General Discussion}

Constraints associated with bimanual coordination arise at many levels (Kelso, 1995). While much of this work has focused on biomechanical factors (e.g., activation of homologous muscles), recent studies have pointed to the importance of higher level variables such as attention (Pellecchia \& Turvey, 2001) and goal-based representations (Fink et al., 2000; Mechsner et al., 2001). Defining what is meant by the term "goal" can be difficult. Moreover, our recent work has shown that subtle changes in the task requirements can have dramatic changes on performance, results interpreted as being the consequence of variation in goalbased representations. For example, the difficulty people have producing orthogonal trajectories in reaching tasks is abolished when the targets are specified directly rather than symbolically (Diedrichsen, Hazeltine, Kennerly, \& Ivry, 2001). We have hypothesized that directly cued goals are coded as target locations whereas the goals for symbolically-cued goals are coded as movement trajectories (Ivry, Diedrichsen, Spencer, Hazeltine, \& Semjen, 2004). Interference effects are much more pronounced for the spatial codes associated with abstract trajectories.

Similarly, we have hypothesized that goals and their associated control processes are quite different for rhythmic movements that involve discontinuities compared to those that are 
R. M. C. Spencer, R. B. Ivry, E. Hazeltine, \& A. Semjen

produced continuously (Spencer et al., 2003). The discontinuities define salient events and are hypothesized to constitute temporal goals from cycle to cycle. With continuous movements, timing may be emergent, reflecting the operation of different control processes that satisfy the task goals (e.g., angular velocity).

The influence of event-based goals on rhythmic movements has also been emphasized in recent studies by Kelso and colleagues (Fink et al., 2000; Kelso, Fink, DeLaplain, \& Carson, 2001). Measures of relative phase are more stable when a pacing metronome is used to present two beats per cycle compared to when only a single sound is presented each cycle. However, if the metronome alternates with a singular contact point, coordination stability decreases.

Rather than imposing an external metronome to pace the movements in our study, the participants were required to simultaneously vocalize, and we examined the frequency between the manual and vocal responses. We assume that the vocalizations reveal how the participants conceptualize the structure of the task. In particular, we expected that vocalizations would be concurrent with salient events during the hand movements. This procedure allowed us to examine the goal representations underlying bimanual movements with and without real or imaginary events.

The inclusion of the physical boards had a strong influence on the vocalization patterns suggesting that these salient events imposed a goal-based representation of the task. When the contact boards were absent, the vocalizations and movements tended to be uncoupled with the rate of vocalization independent of hand movement rate. We have hypothesized that movements without salient features such as abrupt reversals, pauses, or external contacts, need not rely on the operation of an event-based representation of the temporal goal (Spencer et al., 2003).

It should be noted that while we assume that timing may be emergent during continuous movements, we did not, a priori, anticipate the striking degree of uncoupling in the conditions without the external events in this study. In a unimanual finger flexion/extension task in which participants were instructed to vocalize concurrently, spontaneous synchronization was adopted (Chang \& Hammond, 1987; Kelso, Tuller, \& Harris, 1983; Klapp, 1981). Moreover, in preliminary studies using concurrent spontaneous vocalization during bimanual movements we observed strong coupling, even in the absence of a contact surface (Ivry et al., 2004). In these studies, the hand movements involved flexion/extension about the wrist with the palms facing downward; as such movements in these studies may have been more abrupt due to the effects of gravity. Factors that influence the strength of vocal-manual coupling might include the specific muscles used to produce the movement, hand orientation, and/or movement abruptness; future research will be required to assess this issue.

We expected performance when imagining the presence of the contact points would be similar to when the contact boards were present. However, the results for the imagine-event condition were much more similar to the no-event condition. Two explanations seem viable. First, it is possible that the participants failed to comply with the task instructions, or at least failed to consistently imagine the contact points. Second, imagination of an event may not be 
sufficiently salient to establish a goal-based representation. Participants in this group had no exposure to the event conditions. As such, imagining such a scenario may not be realistic or accurate.

When the boards were present, the event timing, as inferred from the vocalization patterns, was strongly constrained by the location of the boards. Boards located in the center of the hand trajectories resulted in a tendency for the participants to adopt a 1:1 vocal-manual ratio for in-phase movements and a 2:1 ratio for anti-phase movements. When the right hand board was displaced, this pattern was reversed. Thus, the number of vocalizations per cycle was tightly coupled to the number of haptic events in the cycle.

The number of vocalizations may provide an index of the complexity of an event-based representation. If so, these results suggest that the complexity of the in- and anti-phase movement patterns are malleable, and under appropriate conditions, anti-phase movements may become "simpler" than in-phase movements (see also Kelso, 1995; Mechsner et al., 2001).

Many studies of bimanual coordination have involved rhythmic movements without contact surfaces, and a consistent observation is that in-phase movements are more stable than antiphase movements. The standard deviation of relative phase remains constant across movement frequencies and this pattern can be maintained close to maximal movement rates. In contrast, fluctuations in relative phase are found during anti-phase movements as movement rate increases, with occasional transitions to the in-phase mode. This phenomenon has been modeled in terms of coupled oscillators, with the instability at high frequencies for anti-phase movements reflecting the attraction towards a stronger attractor for the in-phase pattern.

We propose an alternative perspective based on representational differences related to the relative event timing for in-phase and anti-phase movements. Movements with abrupt onsets or with haptic feedback from an external contact point (e.g., table tapping) would likely entail a temporal representation similar to that observed in the present study in which both boards were placed in the center. For example, if the movements involve flexion/extension of the index fingers, either against a table surface or in midair, salient events might correspond to the contact point with the table or the pause that people tend to introduce prior to each flexion cycle (Ivry, Spencer, Zelaznik, \& Diedrichsen, 2002). These contacts or pauses would occur simultaneously for the two hands during in-phase movements, and alternate during antiphase movements. Thus, as demonstrated here, anti-phase movements may have a more complex representation (two event goals per cycle) than in-phase movements (one event goal per cycle) in such tasks. It may be difficult to maintain this complexity at fast movement rates. Phase transitions, in this perspective, reflect a shift to the simpler event representation of inphase movements. While the current study suggests ways to probe event timing, the current data sets are insufficient to directly evaluate predictions relating these representations to pattern stability. 
R. M. C. Spencer, R. B. Ivry, E. Hazeltine, \& A. Semjen

\section{REFERENCES}

Byblow, W. D., Carson, R. G., \& Goodman, D. (1994). Expressions of asymmetries and anchoring in bimanual coordination. Human Movement Science, 13, 3-28.

Chang, P., \& Hammond, G. R. (1987). Mutual interactions between speech and finger movements. Journal of Motor Behavior, 19, 265-274.

Diedrichsen, J., Hazeltine, E., Kennerley, S., \& Ivry, R. B. (2001). Moving to directly cued locations abolishes spatial interference during bimanual actions. Psychological Science, $12,493-498$

Domkin, D., Laczko, J., Jaric, S., Johansson, H., \& Latash, M. L. (2002). Structure of joint variability in bimanual pointing tasks. Experimental Brain Research, 143, 11-23.

Eliassen, J. C., Baynes, K., \& Gazzaniga, M. S. (2000). Anterior and posterior callosal contributions to simultaneous bimanual movements of the hand and fingers. Brain, 123, 2501-2511.

Fink, P. W., Foo, P., Jirsa, V. K., \& Kelso, J. A. S. (2000). Local and global stabilization of coordination by sensory information. Experimental Brain Research, 134, 9-20.

Franz, E. A., Eliassen, J. C., Ivry, R. B., \& Gazzaniga, M. S. (1996). Dissociation of spatial and temporal coupling in the bimanual movements of callosotomy patients. Psychological Science, 7, 306-310.

Franz, E. A., Zelaznik, H. N., \& McCabe, G. (1991). Spatial topological constraints in a bimanual task. Acta Psychologia, 77, 137-151.

Haken, H., Kelso, J. A., \& Bunz, H. (1985). A theoretical model of phase transitions in human hand movements. Biological Cybernetics, 51, 347-356.

Ivry R. B., Diedrichsen J., Spencer R. M. C., Hazeltine E., \& Semjen A. (2004). A cognitive neuroscience perspective on bimanual coordination and interference. In S. Swinnen \& J. Duysens (Eds.), Interlimb Coordination (pp. 259-295). Norwell, MA: Kluwer Academic Publishing.

Ivry, R. B., \& Hazeltine, E. (1999). Subcortical locus of temporal coupling in the bimanual movements of callosotomy patients. Human Movement Science, 18, 345-375.

Ivry, R. B., Spencer, R. M. C., Zelaznik, H. N., \& Diedrichsen, J. (2002). The cerebellum and event timing. Annals of the New York Academy of Sciences, 978, 302-317.

Kazennikov, O., Perrig, S., \& Wiesendanger, M. (2002). Kinematics of a coordinated goaldirected bimanual task. Behavioral Brain Research, 134, 83-91. 
Kelso, J. A. S. (1995). Dynamic Patterns. MIT Press.

Kelso, J. A. S., Fink, P. W., DeLaplain, C. R., \& Carson, R. G. (2001). Haptic information stabilizes and destabilizes coordination dynamics. Proceedings of the Royal Society of London, Series B, 268, 1207-1213.

Kelso, J. A. S., Southard, D. L., \& Goodman, D. (1979). On the coordination of two-handed movements. Journal of Experimental Psychology: Human Perception and Performance, 5, 229-238.

Kelso, J. A. S., Tuller, B., \& Harris, K. S. (1983). A "dynamic pattern" perspective on the control and coordination of movement. In P. F. Macneilage (Ed.), The Production of Speech (pp. 137-172). New York: Springer-Verlag.

Kennerley, S., Diedrichsen, J., Hazeltine, E., Semjen, A., \& Ivry, R. B. (2002). Callosotomy patients exhibit temporal uncoupling during continuous bimanual movements. Nature Neuroscience, 5, 376-381.

Klapp, S. T. (1981). Temporal compatibility in dual motor tasks II: Simultaneous articulation and hand movements. Memory \& Cognition, 9, 398-401.

Mechsner, F., Kerzel, D., Knoblich, G., \& Prinz, W. (2001). Perceptual basis of bimanual coordination. Nature, 414, 69-73.

Pellecchia, G. L., \& Turvey, M. T. (2001). Cognitive activity shifts the attractors of bimanual coordination. Journal of Motor Behavior, 33, 9-15.

Perrig, S., Kazennikov, O., \& Wiesendanger, M. (1999). Time structure of a goal-directed bimanual skill and its dependence on task constraints. Behavioral Brain Research, 103, 95104.

Schmidt, R. C., Shaw, B. K., \& Turvey, M. T. (1993). Coupling dynamics in interlimb coordination. Journal of Experimental Psychology: Human Perception and Performance, 19, 397-415.

Serrien, D. J., \& Wiesendanger, M. (2000). Temporal control of a bimanual task in patients with cerebellar dysfunction. Neuropsychologia, 38, 558-565.

Spencer, R. M., Zelaznik, H. N., Diedrichsen, J., \& Ivry, R. B. (2003). Disrupted timing of discontinuous but not continuous movements by cerebellar lesions. Science, 300, 1437 1439.

Tuller, B., \& Kelso, J. A. (1989). Environmentally-specified patterns of movement coordination in normal and split-brain subjects. Experimental Brain Research, 75, 306-316. 
R. M. C. Spencer, R. B. Ivry, E. Hazeltine, \& A. Semjen

\section{Authors' Note}

We dedicate this paper to the memory of Andras Semien. His work has been instrumental in emphasizing the importance of event structure for understanding constraints associated with rhythmic movements.

We are grateful to Stephanie Yang for her assistance in collecting the data. This work was supported by NIH grants NS30256, NS17778, and NS40813.

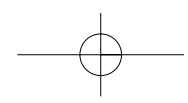

